A N N A L E S Annales de Bretagne et des Pays de l'Ouest

BRETAGNE PAYS DE LOUEST

Anjou. Maine. Poitou-Charente. Touraine

125-1 | 2018

Tolérance et intolérance des religions en Europe, $X V I^{e_{-}}$ XVIII siècle

\title{
De la tolérance à la liberté de religion : les pouvoirs face à la question protestante, France, 1685-1791
}

From toleration to freedom of religion: the authorities and the Protestant question in France, 1685-1791

\section{Luc Daireaux}

\section{(2) OpenEdition} Journals

\section{Édition électronique}

URL : http://journals.openedition.org/abpo/3772

DOI : $10.4000 /$ abpo.3772

ISBN : 978-2-7535-7536-3

ISSN : 2108-6443

Éditeur

Presses universitaires de Rennes

Édition imprimée

Date de publication : 30 mars 2018

Pagination : $59-70$

ISBN : 978-2-7535-7491-5

ISSN : 0399-0826

Référence électronique

Luc Daireaux, «De la tolérance à la liberté de religion : les pouvoirs face à la question protestante, France, 1685-1791 », Annales de Bretagne et des Pays de l'Ouest [En ligne], 125-1 | 2018, mis en ligne le 30 mars 2020, consulté le 06 janvier 2021. URL : http://journals.openedition.org/abpo/3772 ; DOI : https://doi.org/10.4000/abpo.3772 


\title{
De la tolérance à la liberté de religion : les pouvoirs face à la question protestante, France, 1685-1791
}

\author{
LUc DAIREAUX
}

Docteur en histoire, chercheur associé au HisTeMé, université de Caen (EA 7455)

Pour bien comprendre la notion de tolérance telle qu'elle s'exprime dans les textes des $\mathrm{XVII}^{\mathrm{e}}$ et $\mathrm{XVIII}^{\mathrm{e}}$ siècles, il faut nécessairement faire un détour par le lexique et ses évolutions. Le terme est utilisé de manière très restrictive au tournant du Grand Siècle. Il suffit de rappeler la définition proposée par Antoine Furetière dans son Dictionnaire publié de manière posthume en 1690 : « patience par laquelle on souffre, on dissimule quelque $\operatorname{chose}^{1}$ ». En 1702, cette définition est reprise et enrichie par le juriste normand exilé aux Provinces Unies, Henri Basnage : "Le mot de tolérance enferme en soi une condamnation tacite de la chose tolérée : elle signifie un support charitable et pacifique de ce que l'on n'approuve pas ${ }^{2}$. " À la fin du siècle des Lumières, le terme n'a pas encore la charge positive qu'il revêt aujourd'hui. Dans un discours prononcé à l'Assemblée constituante le 7 mai 1791, Charles Maurice de Talleyrand, député, ancien évêque d'Autun, désormais administrateur du département de Paris, déclare ainsi que " la liberté doit être illimitée en fait d'opinions religieuses ". Il refuse de se limiter à une "tolérance hypocrite, qui vouloit bien permettre de penser mais qui ne permettoit pas l'expression de la pensée par des actes publics ». Un peu plus haut, Talleyrand exhortait ses collègues : " Il est tems que l'on sçache que l'expression publique des opinions religieuses ne fait point en vain partie des droits de l'homme, et ne nommons pas tolérance la garantie de cette liberté ; bannissons de la langue cette expression dominatrice ${ }^{3}$."

1. FuRETIÈRE, Antoine, Dictionnaire universel, contenant généralement tous les mots françois tant vieux que modernes et les termes des sciences et des arts..., La Haye et Rotterdam, Arnout et Reinier Leers, 1690, t. 3, s.v. "Tolérance".

2. FuRETIÈRE, Antoine et BASNAge, Henri, Dictionnaire universel..., La Haye et Rotterdam, Arnout et Reinier Leers, 1702, t. 2, p. 979 (souligné dans le texte).

3. Journal politique, ou gazette des gazettes, 20 mai 1791, n ${ }^{\circ} 15$, ici p. 38 (souligné par l'auteur). 
Dès la fin du XVII ${ }^{\mathrm{e}}$ siècle, lexicologues et penseurs distinguent " la tolérance civile de la tolérance ecclésiastique ». Comme l'affirme Henri Basnage dans sa version révisée du Dictionnaire d'Antoine Furetière, la seconde " va à souffrir dans l'Église des sentimens différens et opposez ", tandis que la première cherche à " les suporter seulement dans la société civile ${ }^{4}$ ". Cette tolérance, dite aussi " politique ", " emporte le droit de jouir du bénéfice des loix et de tous les privilèges de la société, sans raport à la différence de religion ". Basnage fait explicitement référence à l'œuvre du juriste et philosophe allemand Samuel von Pufendorf, penseur du droit naturel, auteur d'un De habitu religionis christianae ad vitam civilem (1687), livre qui fait l'objet d'un compte rendu dans l'Histoire des ouvrages des savants dès sa parution et qui est traduit en français en $1690^{5}$.

Le concept de "tolérance civile " - le seul qui nous intéresse ici - est abondamment travaillé et discuté par historiens et philosophes lors des deux dernières décennies du XVII ${ }^{\mathrm{e}}$ siècle. La réflexion se poursuit tout au long du siècle des Lumières à une échelle européenne. Avec Jean Barbeyrac, juriste d'origine languedocienne, traducteur de Pufendorf, auteur d'un Traité de la morale des Pères de l'Église (1728), on trouve "l'affirmation nette et sans ambages du principe de la tolérance civile ${ }^{6}$ ". Celui qui est alors professeur de droit à l'université de Groningue, aux Provinces Unies, peut ainsi déclarer :

"Je conclus qu'un souverain peut à la vérité rendre dominante en quelque façon la religion qui lui paroît la meilleure, et qu'il doit même travailler à sa propagation par toutes les voies légitimes qu'il a en main, mais qu'il ne sauroit légitimement ni forcer les consciences, ni priver quelques-uns de ses sujets, sous prétexte de non-conformité avec la religion dominante, des droits qu'ils ont d'ailleurs en tant qu'hommes et en tant que citoiens ${ }^{7}$."

Il ne s'agit pas de revenir ici sur la genèse et l'épanouissement d'une idée - l'historiographie du sujet n'a cessé de se développer ces dernières années $^{8}$ - mais plutôt de considérer le point de vue des autorités législatives et judiciaires françaises ainsi que le chemin, particulièrement sinueux, qui conduit au triomphe de la liberté de religion, dans le droit et la pratique, au tournant des années 1780-1790.

4. Furetière, Antoine et Basnage, Henri, Dictionnaire universel..., 1702, t. 2, p. 979.

5. Voir l'Histoire des ouvrages des savants, la revue publiée par Henri Basnage à Rotterdam, sept. 1687, p. 267-271, et PuFENDORF, Samuel, Traité de la religion chrétienne par rapport à la vie civile, où l'on voit que l'Église n'est point un État et que la puissance des princes ne va pas jusqu'à dominer sur la foy, Utrecht, Antoine Schouten, 1690 [texte traduit du latin par Monsieur de Saint-Amant, alias Antoine Teissier].

6. C'est ainsi que PuAux, Franck, après avoir évoqué les figures d'Henri Basnage, de Pierre Bayle ou de Denis Papin, conclut Les Précurseurs français de la tolérance au XVII siècle, Paris, Fischbacher, 1881, p. 163.

7. BARBEYRAC, Jean, Traité de la morale des Pères de l'Église..., Amsterdam, Herman Uytwerf, 1728, p. 184.

8. On peut notamment citer la synthèse de Zagorin, Perez, How the Idea of Religious Toleration Came to the West, Princeton, Princeton University Press, 2003. 


\section{Quelle condition juridique pour les protestants après 1685?}

En octobre 1685, au terme d'une longue séquence de persécutions judiciaires, l'édit de révocation est signé à Fontainebleau. Tout en interdisant la pratique publique du culte réformé, il semble ménager une forme de devotio privata :

"Pourront au surplus lesdits de la R.P.R. [Religion prétendue réformée], en attendant qu'il plaise à Dieu les éclairer comme les autres, demeurer dans les villes et lieux de notre royaume, pays et terres de nostre obéissance, et y continuer leur commerce, et jouir de leurs biens, sans pouvoir estre troublez ny empeschez, sous prétexte de ladite R.P.R., à condition, comme dit est, de ne point faire d'exercice, ny de s'assembler, sous prétexte de prières ou de culte de ladite religion, de quelque nature qu'il soit, sous les peines cy-dessus de confiscation de corps et de biens ${ }^{9}$."

Si ce douzième et dernier article apparaît très vite, dans le cortège de violences et de dragonnades qui s'abat sur le pays, comme une "duperie $^{10}$ ", il n'en reste pas moins que l'existence juridique des protestants est réaffirmée. Ceux qui refusent, malgré toutes les pressions, d'abjurer leur foi sont souvent qualifiés d'" opiniâtres ", même si on préfère oublier leur existence, jusqu'à décider de leur expulsion en 1687-1688 ${ }^{11}$. La fiction d'un royaume purement catholique n'est entérinée par le droit qu'en 1715, à quelques mois de la mort du " grand Roi ". La déclaration du 8 mars affirme ainsi sans détour :

« Le séjour que ceux qui ont été de la Religion prétendue réformée, ou qui sont nés de parens religionaires, ont fait dans notre royaume depuis que nous y avons aboli tout exercice de ladite religion, est une preuve plus que suffisante qu'ils ont embrassé la Religion catholique, apostolique et romaine, sans quoi ils n'y auroient pas été soufferts et tolérés ${ }^{12}$."

La déclaration de 1715 admet bien " une présomption de droit : “il n'y a plus de protestants en France" 13 ". Tous les réformés sont donc supposés avoir abjuré leur ancienne foi, " qu'ils aient fait abjuration, ou non, ou

9. Édit du roy, portant défenses de faire aucun exercice publicq de la Religion prétendue réformé[e] dans son royaume. Registré en la chambre des vacations, le 22 octobre 1685, Paris, Jacques Langlois, 1685, p. 7 (article 12).

10. Labrousse, Élisabeth., "Une foi, une loi, un roi? " La révocation de l'édit de Nantes, Paris et Genève, Payot et Labor et Fides, 1985, p. 199.

11. Je me permets de renvoyer à DAIREAUX, Luc, "Les "opiniâtres" de la Révocation : autour d'une expulsion (1688) ", dans Poutrin, Isabelle et TALLON, Alain (dir.), Les Expulsions de minorités religieuses dans l'Europe des XIII -XVII siècles, Pompignac, éditions Bière, 2015, p. 153-173.

12. Voir la déclaration du 8 mars 1715 , dans Nouveau recueil des édits et déclarations, arrêts et règlemens du Conseil, rendus au sujet des gens de la Religion prétendue réformée, Grenoble, André Faure, 1752, p. 326-328, ici p. 327.

13. ANGER, Henry, De la condition juridique des protestants après la révocation de l'édit de Nantes, Paris, Arthur Rousseau, 1903, p. 52. Le concept de " présomption de droit " appliqué à la situation des protestants après 1715 est utilisé abondamment par MALESHERBES, C. G. de, dans son Mémoire sur le mariage des protestans en 1785, s.l.n.d., p. 13, 77 ou 105, et dans le Second mémoire sur le mariage des protestans, Londres, 1787, p. 3 sq. 
que les actes n'en puissent être rapportés ". S'ils refusent " aux curés de recevoir les sacremens ", ils tombent donc sous le coup de la législation concernant les "relaps", un ensemble de dispositions particulièrement sévères ${ }^{14}$. Le texte du 8 mars 1715 suscite un émoi compréhensible chez les magistrats. Ainsi, le procureur général du parlement de Paris, Henri François d'Aguesseau, dans une lettre datée du 26 mars, rappelle fort justement que " le roy a bien aboli l'exercice de la R.P.R. par ses édits " mais qu'il " n'a point ordonné précisément aux religionnaires de faire abjuration et d'embrasser la relligion catholique ". Être ou demeurer protestant ne ressort donc pas de la loi. La rigueur de celle-ci est plutôt " tombée sur les relaps ", un " crime " dont il faut nécessairement apporter la preuve. Henri d'Aguesseau propose donc, en cas d'absence d'acte d'abjuration, " qu'il y soit suppléé par les preuves qui seront rapportées pour établir qu'ils ont exercé la religion catholique et en ont fait les actes ordinaires ${ }^{15}$ ". L'idée du procureur général - et futur chancelier (à partir de 1717) - n'est toutefois pas retenue. Dès le 10 avril 1715, le texte royal figure dans les registres du parlement de Paris.

La déclaration du 14 mai 1724, donnée au sortir de la Régence, très sévère contre les protestants " peu sincèrement réunis à la Religion catholique, apostolique et romaine ", ne reprend pas les formulations de 1715. L'interdiction de tout " exercice de religion autre que ladite religion catholique " est cependant renouvelée, l'obligation du baptême " dans les Églises des paroisses où ils [les nouveaux convertis] demeurent " est également rappelée. Si les différentes mesures répressives qui scandent le texte (contre les prédicants, contre les "relaps ", etc.) s'inscrivent bien dans la fiction d'une France entièrement catholique, la "R.P.R. ", certes renvoyée à une période révolue, est systématiquement opposée à la religion catholique, selon un diptyque confessionnel finalement classique. Le texte demeure particulièrement embarrassé pour nommer ceux qu'on veut punir ou ce qu'ils représentent : "l'hérésie ", les "sujets qui ont ci-devant fait profession de la R.P.R. ", les " religionnaires ${ }^{16}$ ". Aussi ambiguë dans ses formulations soit-elle, la déclaration de 1724, rapidement enregistrée par les parlements du royaume, connaît une large diffusion ${ }^{17}$, ce qui n'implique pas nécessairement une accélération de la répression ${ }^{18}$.

14. Déclaration du 8 mars 1715, dans Nouveau recueil..., p. 326-328.

15. Lettre d'Henri d'Aguesseau, 26 mars 1715, citée, sans son destinataire, d'après BnF, fr. 7046, fol. 38, dans Hugues, Edmond, Histoire de la restauration du protestantisme en France au XVIII siècle - Antoine Court-d'après des documents inédits, Paris, Grassart, 1875, t. $1^{\mathrm{er}}$, p. $367-369$, ici p. $368-369$.

16. Déclaration du roy, concernant la religion, donnée à Versailles le 14 may 1724, Paris, Louis-Denis Delatour et Pierre Simon, 1724, passim.

17. Le texte est enregistré à Paris le 31 mai 1724, à Grenoble le 16 juin, à Pau le 21, à Toulouse le 23, à Rouen le 27, à Douai le 30, etc. Je m'appuie ici sur les éditions imprimées de la déclaration de 1724, retrouvées dans divers catalogues de bibliothèques.

18. Voir KRUMENACKER, Yves, "L'application de la législation antiprotestante en France après 1685 ", dans GARNOT, Benoît, Normes juridiques et pratiques judiciaires du Moyen 


\section{Le pouvoir royal face à un double défi : les sépultures et les mariages protestants}

Si le protestantisme n'a plus d'existence légale, les protestants - au sens large du terme, soit des " opiniâtres " aux " nouveaux convertis " de façade demeurent. Le pouvoir royal et les autorités judiciaires du royaume sont confrontés à deux questions particulièrement épineuses : celle des sépultures et celle des mariages. C'est notamment par ce biais que la question de la tolérance civile est posée tout au long du siècle des Lumières.

Il faut de nouveau rappeler que l'édit de révocation reste particulièrement ambigu. L'article 8 précise que " les enfants qui naîtront de ceux de ladite R.P.R. " seront " dorénavant baptisez par les curés des paroisses ${ }^{19}$ ". Rien n'est dit des mariages et des sépultures. La déclaration du 9 avril 1736, qui reprend les suggestions d'un mémoire de $1732^{20}$, règle le problème des enterrements. L'article 13 de ce texte dispose en effet que l'inhumation de " ceux auxquels la sépulture ecclésiastique ne sera pas accordée " n'interviendra " qu'en vertu d'une ordonnance du juge de police des lieux, rendue sur les conclusions [du] procureur [royal] ou de celui des hauts-justiciers $^{21}$ ". Malgré une formulation alambiquée, le pouvoir royal d'une part reconnaît l'existence de protestants et d'autre part établit une procédure civile qui revient, pour partie en tout cas, sur la législation concernant les " relaps ». La procédure mise au point en 1736 est appliquée et largement documentée ${ }^{22}$.

C'est la question des mariages qui suscite les débats les plus fournis. Dès 1693, l'intendant de la généralité de Caen, Nicolas Joseph Foucault, constate que « la plupart des religionnaires qui ont fait abjuration ne pouvant se marier à l'église, les curés refusant de leur administrer le sacrement de mariage s'ils ne font le devoir de catholiques romains, ils se faisoient des promesses de mariage, sur la foi desquelles ils habitoient ensemble". Et l'intendant de conclure qu'il " arrivera que ces mariages seront déclarés clandestins " et de regretter qu'on n'ait " point pourvu " à ce " désordre ${ }^{23}$ ". Le 15 juin 1697, le préambule de la déclaration donnée à Versailles par Louis XIV fait état de mariages contractés par un « nombre considérable de

Âge à l'époque contemporaine, Dijon, Éditions universitaires de Dijon, 2007, p. 141-150, ici p. 144-147 ("Un texte à ne pas appliquer? La déclaration du 14 mai 1724 »).

19. Édit du roy, portant défenses, op. cit., p. 6.

20. Voir RulHiErRE, C. C. de, EEuvres, Paris, Ménard et Desenne, 1819, p. 474-475 (extrait des Éclaircissements historiques sur les causes de la révocation de l'édit de Nantes...).

21. Déclaration du roy, concernant la forme de tenir les registres des baptesmes, mariages, sépultures, vestures, noviciats et professions, et des extraits qui en doivent estre délivrez, donnée à Versailles le 9 avril 1736, Paris, Imprimerie royale, 1736, p. 7.

22. On peut notamment lire ThIBAUT-PAYEN, Jacqueline, Les Morts, l'Église et l'État dans le ressort du parlement de Paris aux XVII et XVIII siècles, Paris, Fernand Lanore, 1977, p. 189 $s q$, et Molinier-Potencier, Sophie, La Sépulture des protestants de l'édit de Fontainebleau à l'édit de Tolérance (1685-1792), thèse tapuscrite, université Paris 2, 1996.

23. BAUDRY, Frédéric (éd.), Mémoires de Nicolas Joseph Foucault, Paris, Imprimerie impériale, 1862, p. 298. 
personnes " devant des " prêtres autres que leurs propres curés ". Comment imaginer que des actes produits par les notaires puissent " conférer la grâce du sacrement de mariage et suppléer à la bénédiction des prêtres ${ }^{24}$ " ? En cette fin du XVII ${ }^{\mathrm{e}}$ siècle, le pouvoir royal semble en pleine confusion. Deux lignes s'opposent, l'une se contentant de réprimer les manifestations publiques de l'“ hérésie ", l'autre souhaitant aller jusqu'à contrôler la sphère privée ${ }^{25}$. Une consultation secrète est lancée auprès d'intendants et d'évêques. La déclaration du 13 décembre 1698 conclut cette séquence. Si tous les Français sont exhortés à se rendre à la messe, l'idée de contrainte est abandonnée. Il est simplement demandé aux sujets du roi "réunis à l'Église " d'observer " les solennités prescrites par les saints canons ", le roi se réservant de juger les contestations portant sur les effets civils des mariages célébrés depuis $1685^{26}$. Des instructions envoyées aux intendants le 7 janvier 1699 refusent toute forme de « contrainte " contre les « nouveaux convertis " " pour les porter à recevoir les sacrements ${ }^{27}$ ".

Malgré les proclamations de la déclaration de 1724 qui rappelle les lois de 1697 et de 1698, le pouvoir royal ne peut empêcher la multiplication d'unions contractées de manière illégale : mariages de subterfuge supposant des curés délivrant des certificats sans épreuves ou actes de catholicité, unions de fait prenant des formes laïques et surtout mariages célébrés au Désert, bientôt " seuls valables au regard de la norme religieuse " mais « radicalement nuls au regard de la norme juridique ${ }^{28}$ ". Dans l'ancien droit, le concubinage relève du délit pénal et implique des effets civils redoutables, notamment l'incapacité de transmettre des biens à des enfants réputés bâtards. Dans le cas qui nous intéresse, l'illégitimité est provoquée par le pouvoir lui-même, lequel fait de l'abjuration la condition nécessaire au mariage. Toutefois, la question ne trouve une issue judiciaire que quarante après la promulgation de l'édit de Fontainebleau. En effet, les couples mariés avant 1685 conservent leur qualité d'époux légitimes. C'est au décès de leurs enfants, mariés cette fois de manière illicite, que les tribunaux sont sollicités pour répondre à des enjeux de succession $^{29}$.

24. Déclaration du roy, concernant les mariages faits par d'autres prestres que les curez des contractans, donnée à Versailles le 15 juin 1697, Paris, Louis Denis Delatour et Pierre Simon, 1720.

25. Je me contente de reprendre l'analyse de GACHON, Paul, « Le Conseil royal et les protestants en 1698 : l'enquête, la question de la messe et le rôle de Bâville ", Revue historique, t. 85,1904 , p. $252-270$, ici p. 264.

26. Article 7 de la déclaration du 13 décembre 1698, texte publié par LEMOINE, Jean (éd.), Mémoires des évêques de France sur la conduite à tenir à l'égard des réformés (1698), Paris, A. Picard et fils, 1902, ici p. 387.

27. Mémoire du roi " pour servir d'instruction aux intendants ", 7 janvier 1699, texte publié dans ibid., ici p. 394, d'après BnF, fr. 7045, fol. 229 (papiers Rulhière).

28. CARBONNIER, Jean, "L'amour sans la loi : réflexions de psychologie sociale sur le droit de la filiation, en marge de l'histoire du protestantisme français ", Bulletin de la Société de l'histoire du protestantisme français, t. 125, 1979, p. 47-75, ici p. 57.

29. Ibid., p. 57-61. 
L'exemple du Languedoc peut être évoqué. Les premières condamnations d'importance dans la région interviennent en 1739 avec une décision du présidial de Nîmes qui, tenant les grands jours en Vivarais, sanctionne cinq ou six " particuliers". Ces derniers, "mariés par des ministres ", sont convaincus d'avoir vécu " en concubinage public et notoire avec leurs prétendues femmes " et condamnés à une "aumône envers les pauvres " ainsi qu'à une " amende envers le roi ". Les accusés sont invités à cesser d'habiter avec leurs " prétendues femmes " et à solliciter l'évêque diocésain pour obtenir la bénédiction de leurs mariages ${ }^{30}$. La procédure criminelle jugée à Privas semble aller à l'encontre des directives du gouvernement, toujours très prudent sur le sujet ${ }^{31}$. Le parlement de Toulouse, quant à lui, s'attaque aux mariages de subterfuge à partir de 1743. Le 10 mai de cette année, la cour languedocienne " octroie " à son procureur général " acte de l'appel comme d'abus par lui interjetté de la célébration " de mariages de " certains nouveaux convertis du diocèse de Lavaur, sans publication de bans, sans permission de l'ordinaire de leur diocèse, ni du curé de leur paroisse ". Le curé de Camarade et un ancien vicaire de Mérigon, deux localités situées dans le diocèse de Rieux, sont mis en cause ${ }^{32}$. Des mariages qu'ils ont célébrés sont déclarés « non valablement contractés" les 8 août 1743 et 2 mars 1744. L'arrêt de 1744 est connu dans le détail : il interdit à six couples de "cohabiter ensemble". Une procédure est bientôt lancée contre les deux ecclésiastiques et les "prétendus conjoints ${ }^{33}$ ".

Ailleurs dans le royaume, les années 1740 marquent aussi l'avènement d'une offensive contre les mariages contractés de manière illégale. Ainsi, le 21 mai 1749, le parlement de Bordeaux enjoint à 44 personnes, "prétendus mariés et mariées dans les assemblées et prêches, et ailleurs, par des ministres ou prêtres étrangers, et autres que leurs propres curés ", de " se séparer incontinent après la publication du présent arrêt ". Le jugement vaut pour tous les " particuliers de l'un et de l'autre sexe du ressort de la cour, qui se prétendent avoir été ainsi mariés ou fiancés ". Les mariages au Désert - ils concernent ici 30 personnes, dont on demande l'emprisonnement - favorisent les " concubinages " et la bâtardise, est-il ajouté. Les enfants issus de telles unions sont donc « incapables de toutes successions, tant directes que collatérales, et de tous autres effets civils et préroga-

30. Voir GuYOT, Joseph Nicolas, Répertoire universel et raisonné de jurisprudence civile, criminelle, canonique et bénéficiale..., Paris, Visse, 1785, t. 15, p. 105 (s.v. "Religionnaires "), et AzÉmA, Ludovic, La Politique religieuse du parlement de Toulouse sous le règne de Louis XV, Aix-en-Provence, Presses universitaires d'Aix-Marseille, 2010, p. 83-84.

31. C'est ce qu'affirme, au contraire de J.-N. Guyot, RulhiÈRE, C. C. de, Euvres, op. cit., p. 285, qui publie une lettre du duc de La Vrillière aux juges de Nîmes (23 septembre 1739).

32. Arrêt du parlement de Languedoc, 10 mai 1743, texte publié dans Recueil des édits, déclarations et ordonnances du roi, arrêts du Conseil, du parlement de Toulouse..., Toulouse, J. Dupleix, 1784, p. 577-578.

33. Ibid., p. 604 (arrêt du 2 ou 3 mars 1744 et référence à la procédure judiciaire en amont et en aval). Voir aussi AzÉMA, Ludovic, La Politique religieuse..., op. cit., p. 84. 
tives attribués aux enfants légitimes ${ }^{34}$ ". Un arrêt de la même cour, daté du 17 décembre 1749, prononce les peines de galères et d'enfermement contre dix-huit personnes qui n'ont pas respecté la législation sur les mariages. Et l'arrêt de préciser que « les certificats des ministres seroient brûlés par l'exécuteur de la haute-justice à la place du palais de l'Ombrière, en présence des prétendus époux ${ }^{35}$ ". La jurisprudence du parlement de Dauphiné est similaire. Les mariages célébrés au Désert sont condamnés, et les nouveaux convertis doivent verser de lourdes amendes. Selon le chevalier de Beaumont, la cour grenobloise a même " poussé les choses à l'extrémité ", en s'attaquant à plusieurs notaires du ressort, " décrettés de prise de corps pour avoir laissé contracter devant eux des gens qui ne leur avoient pu rapporter des certificats de catholicisme ${ }^{36}$ ".

En ce milieu du XVIII ${ }^{\mathrm{e}}$ siècle, l'heure est bien à la répression, particulièrement active en Languedoc. Cela passe notamment par des campagnes de re-baptêmes et un enrôlement dans la milice de protestants mariés au Désert et considérés comme célibataires. Le 17 janvier 1750, une ordonnance s'élève avec vigueur contre les " contraventions " aux édits qui " se sont multipliées en Languedoc par l'artifice des ministres ou prédicants, qui y ont répandu la fausse opinion d'une prétendue tolérance ${ }^{37}$ ".

Il n'en reste pas moins que les parlements - du moins leurs chambres civiles - sont aussi les inventeurs d'une tradition de "tolérance tacite ", laquelle se cristallise au milieu du XVIII ${ }^{\mathrm{e}}$ siècle $^{38}$. Ainsi, le prédicant et futur pasteur sous la Croix Pierre Boudet dit Gautier peut affirmer dès 1749 que "les membres du parlement [de Normandie, où il se trouve alors] sont portés à la tolérance ", avant d'ajouter que les magistrats rouennais ont déclaré "bons et valables les mariages faits et consommés ensuite de pactes et de promesses signés par main de notaire et contrôlés, encore qu'ils n'eussent pas été bénis en face de l’Église ${ }^{39}$ ». Quelques exemples

34. Arrêt du parlement de Guyenne, 21 mai 1749, texte publié par GeLIN, Henri, « Les mariages au Désert et leurs conséquences en Poitou, en 1749 ", Bulletin de la Société de l'histoire du protestantisme français, t. 43, 1894, p. 642-650, ici p. 644-649 (citation, p. 648649).

35. Voir [BEAUMONT, Chevalier de], L'Accord parfait de la nature, de la raison, de la révélation et de la politique, ou traité dans lequel on établit que les voyes de rigueur, en matière de religion, blessent les droits de l'humanité et sont également contraires aux lumières de la raison, à la morale évangélique et au véritable intérêt de l'État, par un gentilhomme de Normandie, ancien capitaine de cavalerie au service de Sa Majesté..., Cologne, Pierre Marteau, 1753, p. 110-111.

36. Ibid., p. 112.

37. Texte cité, d'après Arch. nat., $\mathrm{O}^{1}$ 446, fol. 10-11, par PAPPAS, John, " La répression contre les protestants dans la seconde moitié du siècle d'après les registres de l'Ancien Régime ", Dix-huitième siècle, no 17, 1985, p. 111-128, ici p. 112-113.

38. Voir Malesherbes, C. G. de, Second mémoire, op. cit., p. 1, 4, 41, 28, 160 (" [système de la] tolérance tacite [des magistrats]").

39. Lettre de Pierre Boudet dit Gautier à Antoine Court, 18 novembre 1749, document cité par Waddington, Francis, Le Protestantisme en Normandie depuis la révocation de l'édit de Nantes jusqu'à la fin du dix-huitième siècle (1685-1787), Paris, J.-B. Dumoulin, Rouen, Lebrument, 1862, ici p. 83. 
nous sont connus avec précision. En 1760, le parlement de Rouen juge qu'un enfant " qui est en possession paisible de son état, qui, comme tel, a recueilli la succession de son père et de sa mère, n'est point obligé de rapporter leur acte de célébration de mariage, pour recueillir une succession collatérale $^{40}$ ". En 1769, les magistrats normands prennent une décision de portée similaire : ils déclarent irrecevable une appelante comme d'abus du mariage des parents d'Élisabeth Gervaise. L'appelante souhaitait contester une succession et s'appuyait sur les circonstances du mariage des parents d'Élisabeth, mariage célébré sans curé mais authentifié par deux notaires. Les juges s'appuient sur une " possession de trente années " et les " reconnoissances " mêmes de l'appelante ${ }^{41}$.

Cette jurisprudence libérale est attestée dans d'autres provinces. Le parlement de Languedoc reconnaît également les effets civils d'unions illégalement contractées, et ce dès les années $1750^{42}$. Si un revirement intervient en 1764 dans le sillage de l'affaire Calas, les magistrats reviennent à l'ancienne jurisprudence en $1769^{43}$. En 1770, à Toulouse, l'avocat général Jean-Louis Emmanuel Augustin de Cambon demande qu'on ne " puisse pas troubler [les] enfans dans la possession de leur état " lorsque leurs parents ont vécu et ont été reconnus " comme de légitimes époux ». Et d'ajouter :

" Il ne faut pas se demander à soi-même si l'on est persuadé de l'existence du mariage dont on conteste la vérité, mais il faut se demander si l'intérêt public n'exige pas qu'on le présume. [...] Une expérience malheureuse a fait connoître l'inutilité des moyens dont on s'est servi jusqu'à ce jour pour déraciner l'erreur, et nous ne doutons pas qu'à l'avenir on n'en emploie qui seront plus conformes aux règles de la saine politique et aux loix de l'humanité ${ }^{44}$."

\section{Vers l'édit de 1787 et au-delà}

Durant ce second tiers du XVIII ${ }^{\mathrm{e}}$ siècle, tout semble conduire à un changement législatif. Comme le suggèrent les évolutions jurisprudentielles des parlements, il paraît inconcevable de continuer à vivre dans une espèce de fiction d'une France toute catholique. Pourtant, la condition juridique des protestants n'est modifiée qu'en 1787. L'intransigeance d'une partie non négligeable du clergé et la révérence à l'égard des édits du Roi-Soleil consti-

40. GuYot, Joseph Nicolas, Répertoire universel..., op. cit., t. 15, p. 107 (26 juin 1760).

41. Ibid., p. 107-108 (14 août 1769).

42. AzÉMA, Ludovic, La Politique religieuse..., op. cit., p. 87 (arrêts des 12 septembre 1753 et 9 mars 1759).

43. Ibid., p. 261 (arrêt du 19 août 1769).

44. Texte cité dans Mémoires et plaidoyers de M. Linguet, avocat à Paris, t. 6, Amsterdam, Simon Joly, 1773, p. 159-161 (citations p. 160, réquisitions du 9 juillet 1770, dans le cadre de l'affaire Étienne Salles). Voir aussi AzÉmA, Ludovic, La Politique religieuse..., op. cit., p. 261-263, et PoumarÈDE, J., " Le combat des juges et des avocats pour l'état civil des protestants, ou les ambivalences du droit ", dans La Tolérance, République de l'esprit. Actes du colloque Liberté de conscience, conscience des libertés, tenu à Toulouse du 26 au 28 novembre 1987, Paris, Les Bergers et les mages, 1988, p. 106-116, ici p. 112. 
tuent les principaux facteurs d'explication de l'attentisme gouvernemental. Voltaire le résume en quelques mots bien sentis en 1772, lorsqu'il déclare, dans ses réflexions Sur le procès de Mlle Camp, du nom d'une Montalbanaise, abandonnée par son mari devenu catholique, mari finalement condamné à verser une forte somme à sa femme, alors même que l'union n'était pas reconnue : "On doit espérer qu'un jour la sagesse du ministère trouvera le moyen de concilier ce qu'on doit à la religion dominante et à la mémoire de Louis XIV avec ce qu'on doit à la nature et au bien de la patrie ${ }^{45}$."

Le pouvoir royal est pourtant bien conscient du problème. Dès 1726 , le cardinal Fleury est sensible à l'idée de l'abbé Robert, prêtre à Nîmes, favorable à l'établissement de deux mariages, l'un pour les catholiques, l'autre pour les protestants. Le second est envisagé comme un simple « engagement pris par les conjoints et béni par le curé avec l'eau et le signe de la croix, qui, sans être sacrement, auroit cependant tous les effets civils ${ }^{46}$ ". Ce projet est finalement abandonné. Des avis sont sollicités par Louis XV et ses ministres. On peut citer le rapport de Guillaume François Joly de Fleury, procureur général du parlement de Paris (1752) et celui de Pierre Gilbert des Voisins, conseiller d'État $(1767)^{47}$. Ce dernier avis comprend un projet de déclaration royale octroyant une liberté de culte limitée à la sphère domestique et imaginant une procédure civile d'enregistrement des mariages $^{48}$. Tout le travail de Malesherbes s'inscrit donc dans une dynamique initiée sous le règne de Louis XV.

Sans revenir en détail ici sur la genèse et les caractéristiques de l'édit de Versailles (novembre 1787) ${ }^{49}$, il convient de rappeler que ce texte établit les conditions d'exercice d'une tolérance civile à l'égard des non catholiques. Le terme de " protestants " n'apparaît qu'une seule fois, dans le préambule de l'édit. La liberté de conscience reste implicite, le culte public est interdit. Pour les non catholiques, un état civil est mis en place afin d'enregistrer les naissances, les mariages et les décès. Les publications des mariages seront faites soit par les curés ou les vicaires, soit par les officiers de justice. Des couples mariés au Désert ont la possibilité de légaliser leur union ${ }^{50}$.

45. VolTAIRE, Euvres complètes, Société littéraire typographique, 1785, t. 30, p. 501 (texte initialement paru en 1772).

46. Malesherbes, C. G. de, Mémoire, op. cit., p. 137.

47. Grosclaude, Pierre, Malesherbes, témoin et interprète de son temps..., Paris, Fischbacher, 1961, p. 355 sq.

48. Mémoires sur les moyens de donner aux protestans un état civil en France, composé de l'ordre du roi Louis XV par feu M. Gilbert de Voisins, conseiller d'État, 1787, ici p. 135 sq.

49. L'expression d'" édit de tolérance " semble rarement utilisée avant le troisième tiers du XIX ${ }^{\mathrm{e}}$ siècle. Le centenaire de 1887 paraît marquer une rupture. Voir Viguí, Ariste, "Histoire de la liberté religieuse en France : le premier centenaire de l'édit de tolérance ", Revue politique et littéraire. Revue bleue, $2^{\mathrm{e}}$ semestre $1887, \mathrm{n}^{\mathrm{0}} 21,19$ novembre 1887 , p. 642-650.

50. Édit du roi, concernant ceux qui ne font pas profession de la religion catholique, Lyon, Imprimerie du roi, 1788. Voir aussi LEFEBVRE-TEILLARD, Anne, "Les problèmes juridiques posés par l'édit de 1787 ", et DuBIEF, Henri, " La réception de l'édit du 17 novembre 1787 
Avec la Révolution, on change évidemment de paradigme. La notion de tolérance finit par ne plus avoir de sens dans un monde où priment désormais les droits individuels. L'article 10 de la Déclaration des droits de l'homme et du citoyen, voté le 23 août 1789, demeure limitatif : " Nul ne peut être inquiété pour ses opinions, même religieuses, pourvu que leur manifestation ne trouble pas l'ordre public établi par la loi. " La liberté de culte n'est pas établie formellement et demeure suspendue à la notion d'ordre public. La question fait l'objet d'un âpre et substantiel débat à l'Assemblée constituante, débat au cours duquel Mirabeau et Rabaut Saint-Étienne donnent toute la mesure de leur talent. Le 22 août, le comte de Mirabeau peut ainsi déclarer :

" Je ne viens pas prêcher la tolérance. La liberté la plus illimitée de religion est à mes yeux un droit si sacré que le mot tolérance, qui essaye de l'exprimer, me paraît en quelque sorte tyrannique lui-même, puisque l'existence de l'autorité qui a le pouvoir de tolérer attente à la liberté de penser, par cela même qu'elle tolère et qu'ainsi elle pourrait ne pas tolérer ${ }^{51}$. "

Le lendemain, 23 août, Rabaut Saint-Étienne, qui s'est illustré dans le combat pour l'avènement de l'édit de 1787, rejette pareillement l'usage d'un terme devenu caduque :

" La tolérance! Je demande qu'il soit proscrit à son tour, et il le sera, ce mot injuste, qui ne nous présente que comme des citoyens dignes de pitié, comme des coupables auxquels on pardonne, ceux que le hasard souvent et l'éducation ont amenés à penser d'une autre manière que nous ${ }^{52}$. "

L'heure est maintenant à la liberté de religion et à l'égalité entre les citoyens. La Constitution de 1791 marque un tournant. Son titre premier accorde en effet " la liberté à tout homme de parler, d'écrire, d'imprimer et publier ses pensées, sans que les écrits puissent être soumis à aucune censure ni inspection avant leur publication, et d'exercer le culte religieux auquel il est attaché ${ }^{53}$ ".

L'histoire de la tolérance, vue du point de vue du pouvoir royal et des autorités judiciaires dans la France du XVIII ${ }^{\mathrm{e}}$ siècle, demeure donc celle d'un immense paradoxe. Face à la résistance protestante et à la question cruciale des mariages et des enterrements de ceux qu'on appelle les "religionnaires ", le gouvernement préfère ne rien voir puis proclame une France toute catholique, avant de chercher, sans vraiment le reconnaître, à imaginer des solutions nouvelles. L'édit de 1787 vient très tardivement et dit tout

par les parlements ", Bulletin de la Société de l'histoire du protestantisme français, t. 134, 1988, p. 241-259 et 281-295.

51. Archives parlementaires de 1787 à 1860, $1^{\text {re }}$ série (1789-1799), Paris, Paul Dupont, 1875 , t. 8 , p. 473.

52. Ibid., p. 479. Voir aussi DuPONT, André, Rabaut Saint-Étienne, 1743-1793. Un protestant défenseur de la liberté religieuse, Genève, Labor et Fides, 1989, p. 82-85.

53. Texte cité d'après l'édition en ligne proposée par le Conseil constitutionnel, [http:// www.conseil-constitutionnel.fr] [4 février 2017]. 
autant la place qu'a su conserver le protestantisme malgré la Révocation et les persécutions que le malaise du pouvoir royal face à la diversité religieuse. La rupture paradigmatique que constitue la Révolution, deux ans plus tard, renvoie, du point de vue du droit, la tolérance à une époque révolue, sans liberté et sans égalité. Ce n'est pourtant pas la fin d'un concept. Il faudra encore du temps pour que la tolérance acquière toute la charge positive que le terme porte à l'époque contemporaine, non plus cette fois dans le domaine législatif et politique mais dans le champ de la morale et de la philosophie.

\begin{abstract}
RÉSUMÉ
Il s'agit ici d'interroger la tolérance non dans la seule perspective philosophique mais en termes juridiques et politiques. La communication revient sur le point de vue des autorités législatives et judiciaires françaises ainsi que sur le chemin, parfois sinueux, qui conduit au triomphe de la liberté de religion, dans le droit et la pratique, au tournant des années 1780-1790. Tout au long du $\mathrm{XVIII}^{\mathrm{e}}$ siècle, confronté notamment à la persistance du protestantisme et à la multiplication des mariages et des enterrements illégaux, le pouvoir royal cherche à imaginer des solutions nouvelles, jusqu'au point de proposer un état civil pour les non catholiques, en 1787. La Révolution, sans épuiser le concept, renvoie cependant la tolérance au passé, celle d'une période sans liberté et sans égalité.
\end{abstract}

\title{
ABSTRACT
}

Here, we interrogate tolerance, not only from a philosophical perspective, but also in legal and political terms. The article concentrates on the point of view of French legislative and judicial authorities, and the path, sometimes winding, that lead to the triumph of religious liberty, in right and practice, during the $1780 \mathrm{~s}$ and 90s. Throughout the $18^{\text {th }}$ century, confronted notably by the persistence of Protestantism and the increase in illegal marriages and funerals, the monarchy sought to imagine new solutions, eventually proposing a civil state for non-Catholics in 1787. The Revolution, without exhausting the concept, nevertheless confined tolerance to the past, a period without liberty or equality. 